\title{
VERGABE DES VFI-FÖRDERUNGSPREISES 2015
}

\section{von Otto Oberhauser}

Am 25.01.2016 hat die Vergabekommission des Vereins zur Förderung der Informationswissenschaft (VFI), Wien, entschieden, in diesem Jahr einen mit EUR 300 dotierten Anerkennungspreis für folgende Arbeit zu vergeben:

\section{Anna Knoll (Anna Lamparter): Kompetenzprofil von Information Professio- nals in Unternehmen. Masterarbeit, Hochschule Hannover, 2015.}

Abstract: Information Professionals sind in Unternehmen für den professionellen und strategischen Umgang mit Informationen verantwortlich. Da es keine allgemeingültige Definition für diese Berufsgruppe gibt, wird in der Masterarbeit eine Begriffsbestimmung unternommen. Mit Hilfe dreier Methoden - Auswertung von relevanter Fachliteratur, Untersuchung von einschlägigen Stellenausschreibungen, Führen von Experteninterviews wird ein Kompetenzprofil für Information Professionals erstellt. 16 Kompetenzen in den Bereichen Fach-, Methoden-, Sozial- und persönliche Kompetenzen geben eine Orientierung über vorhandene Fähigkeiten dieser Berufsgruppe für Personalfachleute, Vorgesetzte und Information Professionals selbst.

Urteil der Vergabekommission: Diese Arbeit beinhaltet eine fundierte Untersuchung mit einem interessanten Methoden-Mix, die für Ausbildungsfragen relevant und hilfreich ist.

Der Preis wurde damit zum zehnten und letzten Mal vergeben. Der VFI setzt seine Förderungsarbeit 2016 mit einem neu gegründeten Open Access Journal fort, über das in der nächsten Ausgabe der „Mitteilungen der VÖB" berichtet wird.

Otto Oberhauser (Schriftführer) Verein zur Förderung der Informationswissenschaft (VFI)

E-Mail: oberh@web.de Website: http://www.vfi-online.org

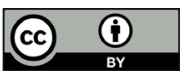

Dieses Werk ist lizenziert unter einer

Creative-Commons-Lizenz Namensnennung 4.0 International 